\title{
The Effects of Insoles for Postural Correction on Spatial-tem- poral Changes of Gait in Spastic Cerebral Palsy Children
}

\begin{abstract}
Improvement in functional gait is one of treatment goals in treatment of cerebral palsy children. This study intended to examine the effects of insoles for postural correction on gait in spastic cerebral palsy patients by investigating changes in gait temporal spatial parameters. As the subjects, 15 spastic bilateral cerebral palsy patients participated in this study. Temporal spatial parameters of gait were measured using GAITRite system under three gait conditions. Bare foot gait, gait in shoes, and gait in insoles for postural correction were conducted. In order to look at differences in temporal spatial parameters according to three gait conditions, repeated one way analysis of variance was conducted. As post hoc test, Bonferroni was conducted. A significant level was set at $\alpha=.05$. According to the result of this study, gait velocity, cadence, step length, stride length of the left lower extremity significantly changed. When the subjects put on customized insoles for postural correction, the effect was greatest. There were no significant changes in stance time, single support time, double support time, swing \% of gait, and stance \% of cycle. Therefore, gait with insoles for postural correction positively influenced functional gait improvement and will be able to be usefully employed for spastic cerebral palsy children as one of gait assistance devices.
\end{abstract}

Key words: Cerebral palsy; Postural insoles; Temporal spatial parameters

\author{
Hee Tak Kima, Sang Wan Lima \\ ${ }^{a}$ Department of Physical Therapy, Pohang \\ College, Pohang, Korea \\ Received : 15 June 2015 \\ Revised : 7 August 2015 \\ Accepted : 18 August 2015 \\ Address for correspondence \\ Sang Wan Lim, PT, MSc. \\ Department of Physical Therapy, \\ Pohang College, Pohang, Korea \\ 60 Sindeok-ro, Heunghae-eup, Buk- \\ gu, Pohang, Korea \\ +82-54-245-1297 \\ windht@pohang.ac.kr
}

\section{INTRODUCTION}

Cerebral palsy is a non progressive lesion of the immature brain under the developmental state of fetuses or infants and triggers disability in movement and postural development(1). In particular, transformation of the musculoskeletal system secondarily triggered, abnormal muscle tone, inappropriate balance and movement control are problems related to gait(2,3). In the case of spastic cerebral palsy, secondary problems trigger abnormality in gait of diverse aspects such as crouch gait or equine gait(4). In addition, pressure is concentrated on the forefoot while supporting weight, which triggers transformation in the foot and causes difficulty with physical balance and weight support of the foot and the ankle joints, resulting in difficulty with gait ability(5).

For gait improvement, foot orthotics have been applied to spastic cerebral palsy children(6, 7).
Orthoses of conventional method are devices applied for prevention of transformation of the foot and ankle and gait stability. Nonetheless, they do not greatly affect muscle activity and changes in kinematic elements required under diverse conditions during gait(8). In addition, occurrence of diverse transformations of the foot does not affect much functional changes such as temporal spatial variables(9). Moreover, applying existing standardized rigid orthoses to cerebral palsy children with spasticity, contracture, and movement disorder implies diverse problems(10).

Postural insole is used in order to improve foot functions or prevent foot transformation for foot alignment and postural stability enhancement during body support in static posture and dynamic posture during gait(11). Insole is used to improve body alignment and prevent or realign transformation. Insole increases stability when increase in the support plane increases as the contact area of 
the foot enlarges and in the level of the foot during weight support. In addition, insole triggers changes in kinematic elements during gait through changes in plantar pressure distribution $(12,13)$. Insole of such form affects proprioceptive sense of the muscles, genearting proprioceptive sense information of positive form. Insole for postural correction customized for individuals, not standardized form, changes tension of postural muscles related to the foot as well as changes in foot pressure(10).

Like this, most research on spastic cerebral palsy patients examined changes in muscle activity and articular angle during gait by applying an assister of hard form and there was almost no research which observed spatial temporal indexes, functional elements of gait evaluation in spastic cerebral palsy children.

Thus, this study examined the effects of changes in spatial-temporal indexes on functional gait during bare foot gait, gait in shoes, and gait in insoles for postural correction and intends to present a gait assist device which may be applied to cerebral palsy patients.

\section{METHODS}

\section{Subjects}

As the subjects, 15 spastic bilateral cerebral palsy patients participated in this study without differentiating males and females. The researcher sufficiently explained the purpose and meaning of the research to the subjects and their guardians and collected data from those who consented to participate in the experiment and had them participate in the research(Table 1). The selection criteria for the subjects are as follows(1). 1)The level of spasticity of the subjects was measured with modified ashworth scale(MAS). As for the level of spasticity, those who were classified as grade 1 and grade 2 were selected. 2)The cerebral palsy children were those who were classified into levels 1 and 2 by gross motor function classification system who were able to independently walk without assistance. 3) Children who had no problem with cognitive level to understand directions were selected as the subjects. 4)The subjects were those who did not receive a surgery or had no pain, damage, or chronic or acute disease during the past six months.
Table 1. General characteristics of the subjects (n=15)

\begin{tabular}{cc}
\hline & Mean \pm Standard deviation \\
\hline Age $(\mathrm{yrs})$ & $11.20 \pm 2.57$ \\
Height $(\mathrm{cm})$ & $133.20 \pm 12.60$ \\
Weight $(\mathrm{kg})$ & $33.15 \pm 11.98$ \\
Foot lenght(mm) & $201.25 \pm 38.95$ \\
Foot width(cm) & $11.25 \pm 2.26$ \\
GMFCS* & $2 \pm 0.66$ \\
MAS+ & $2 \pm 0.52$ \\
\hline
\end{tabular}

*GMFCS: Gross Motor Function Classification System

+MAS: Modified Ashworth Scale

\section{Study Tools and Methods}

\section{Insole for postural correction}

The insoles applied in this study were custom made foot orthotics copying the foot shapes of each subject. They were made with polyurethane by alFOOTs, co., Ltd., Korea which used a high density rebound resilience pad, cupsole for sole arch support, and low elastic band to absorb shock on the heels and polimer gel(Fig. 1).

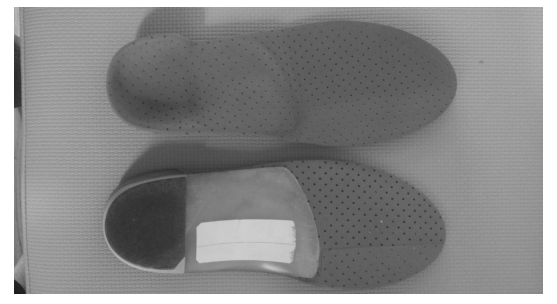

Fig. 1. Postural Insoles

\section{GAITRite system}

In order to examine changes in temporal-spatial index, GAITRite system (GAITRite CIR System Inc., CA, U.S.A, 2010), equipment to collect data by sensing foot pressure during gait, was employed. GAITRite shows the degree of asymmetry of the lower limbs with spatial variables like step length, evaluates lower limb disability with temporal variables and speed like a supporter, and quantify gait patterns after gait intervention; It is equipment with high usability as a clinical evaluation tool(14). GAITRite system is an electronic gait plate whose length and width are $4.5 \mathrm{~m}$ and $0.9 \mathrm{~m}$ and 13,824 sensors whose diameter is $0.6 \mathrm{~cm}$ are vertically arranged at an interval of $1.27 \mathrm{~cm}$ along the foot path and therefore information on spatial temporal variables is collected from this sensor. 
Among the entire length of the gait plate, the length of $3.6 \mathrm{~m}$ and the width of $0.61 \mathrm{~m}$ is an active area where these sensors sense pressure and collects load by the subject's foot at a sampling rate of $90 \mathrm{~Hz}$ per second and sends this information to the computer through a series interface cable. Information about collected temporal-spatial variables was processed with GAITRite system version 3.8 software.

\section{Experimental method}

The experimental method of this study was to take measurement using GAITRite system under the three different conditions below.

1) Gait evaluation with bare foot

2) Gait evaluation with shoes without insoles

3) Gait evaluation with insoles.

The order of evaluating gait was randomly determined. Experimental subjects walked with a comfortable speed staring at the front standing about one meter from GAITRite system for gait evaluation. They walked a total of three times. Measurement was taken after sufficient explanation on how the experimental tool was used.

\section{Data analysis}

Statistical analysis for all collected data was made with SPSS 18.0 for window version. The subjects walked barefoot, walked wearing shoes without insoles, and walked wearing shoes with insoles. In order to look at differences in temporal spatial elements according to three gait conditions, repeated one way analysis of variance was conducted. As post hoc test, Bonferroni was conducted. A significance level $\alpha$ was set at .05.

\section{RESULTS}

Comparison of Temporal-Spatial Indexes according to barefoot gait, gait wearing shoes, and gait wearing insoles for postural correction. Table 2 shows the results of changes in temporal-spatial indexes according to barefoot gait, gait with shoes without insoles, and gait with insoles for postural correction. Gait velocity significantly increased according to different gait conditions( $\mathrm{p}<.05)$.

Table 2. Temporal spatial variables in barefoot, wearing shoes without insoles, and wearing shoes with insoles for postural correction $(n=15)$

\begin{tabular}{cccccc}
\hline & & Barefoot & Shoes without Insoles & Shoes with Insoles & $F$ \\
\hline Velocity(cm/s) & & $66.72 \pm 17.51$ & $74.87 \pm 15.83$ & $85.96 \pm 11.88$ & $10.506^{*}$ \\
Cadence(steps/min) & & $96.54 \pm 14.83$ & $98.55 \pm 14.83$ & $116.51 \pm 10.58$ & $15.073^{*}$ \\
Step length(cm) & R & $37.59 \pm 7.17$ & $42.04 \pm 7.67$ & $45.41 \pm 6.82$ & $16.019^{*}$ \\
& L & $36.49 \pm 5.97$ & $41.62 \pm 6.21$ & $42.97 \pm 6.72$ & $16.915^{*}$ \\
Stride length(cm) & R & $78.64 \pm 11.31$ & $85.31 \pm 10.87$ & $89.38 \pm 12.21$ & 6.123 \\
Stance time(sec) & L & $72.59 \pm 11.38$ & $73.91 \pm 11.72$ & $87.75 \pm 13.44$ & $18.133^{*}$ \\
& $\mathrm{R}$ & $0.55 \pm 0.13$ & $0.55 \pm 0.10$ & $0.57 \pm 0.14$ & 0.714 \\
Single support time(sec) & $\mathrm{L}$ & $0.68 \pm 0.15$ & $0.67 \pm 0.11$ & $0.71 \pm 0.12$ & 0.284 \\
& $\mathrm{~L}$ & $32.76 \pm 4.97$ & $35.46 \pm 3.57$ & $36.27 \pm 3.22$ & 4.449 \\
Double support time(sec) & $\mathrm{R}$ & $35.03 \pm 5.94$ & $35.15 \pm 5.69$ & $35.35 \pm 4.82$ & 0.109 \\
& $\mathrm{~L}$ & $29.71 \pm 11.21$ & $30.33 \pm 10.46$ & $28.06 \pm 5.26$ & 0.600 \\
Swing \% of cycle & $\mathrm{R}$ & $35.65 \pm 6.55$ & $28.53 \pm 5.55$ & $29.51 \pm 8.61$ & 0.473 \\
& $\mathrm{~L}$ & $34.03 \pm 6.21$ & $35.15 \pm 5.81$ & $36.32 \pm 2.59$ & 0.711 \\
Stance \% of cycle & $\mathrm{R}$ & $65.08 \pm 5.18$ & $35.45 \pm 3.43$ & $36.74 \pm 4.39$ & 1.629 \\
& $\mathrm{~L}$ & $65.96 \pm 6.25$ & $64.22 \pm 4.01$ & $64.51 \pm 4.74$ & 0.525 \\
\hline
\end{tabular}

${ }^{*}$ p<0.05 R: Right, L: Left 
According to the result of post-hoc test in accordance with gait conditions, there was significant difference during gait with insoles for postural correction( $\mathrm{p}<.05)$ and gait with shoes and insoles for postural correction( $\mathrm{p}<.05)$. Cadence significantly increased according to different gait conditions $(p<.05)$. According to the result of post-hoc test in accordance with gait conditions, there was significant difference during gait with bare foot, during gait with insoles for postural correction ( $p<.05)$ and gait with shoes and insoles for postural correction $(p<.05)$. Step length of the right lower extremity significantly increased according to different gait conditions(p<.05). According to the result of post-hoc test in accordance with gait conditions, there was significant difference during gait with bare foot and with shoes(p<.05) and during gait with barefoot and insoles for postural correction( $\mathrm{p}<.05)$. Step length of the left lower limbs significantly increased according to different gait conditions(p<.05). According to the result of post-hoc test in accordance with gait conditions, there was significant difference during gait with bare foot and with shoes( $(\mathrm{p}<.05)$ and during gait with shoes and customized assisters(p<.05). Stride length of the right lower limb did not significantly differ according to gait conditions(p $>$.05). Stride length of the left lower limb showed significant difference according to gait conditions( $p<.05)$. According to the result of post-hoc test in accordance with gait conditions, there was significant difference during gait with bare foot and with shoes $(\mathrm{p}<.05)$ and during gait with shoes and insoles for postural correction $(\mathrm{p}<.05)$. There were no significant difference between the left and right lower limbs in stance time, single support time, double support time, swing $\%$ of gait cycle, and stance \% of cycle(p>.05).

\section{DISCUSSION}

Cerebral palsy patients undergo inappropriateness in movement between joints due to transformation of the muscles and the bones, restriction to range of motion, and spasticity $(2,5)$. Therefore, cerebral palsy children exhibit abnormal gait such as crouch gait, scissors gait, and equinus gait(15). Hence, mostly ankle foot orthosis was applied as a means to improve gait problems or functional gait in spastic cerebral palsy children(16). However, the purpose of wearing ankle foot orthosis is to improve ankle range of motion of spastic cerebral palsy children accompanying talipes equinus. Therefore, this study examined changes in spatial temporal elements of gait while walking with barefoot, walking with shoes, and walking with insoles for postural correction in order to improve functional gait. There was significant difference in speed, cadence, step length, and stride length of the left lower limb among spatial temporal elements of gait. However, there was no significant difference in stride length of the right lower limb, stance phase time, single support time, double support time, swing phase rate during gait, and stance phase rate during gait among spatial temporal indexes. Most researchers noted that in spastic cerebral palsy children, analysis of spatial temporal elements of gait was very important(17). In particular, speed, cadence, and stride length as basic elements of gait evaluation were correlated and as one element increased, the other elements linearly increased with positive correlation(18). In addition, cerebral palsy children increased cadence as a strategy to increase speed, thereby improving efficiency of gait(19). In the present study as well, elements of speed, cadence, and stride length significantly increased. In particular, speed and step length had high correlation in functional gait evaluation elements; increase in step length increased speed. In the present study result as well, speed, cadence, step length, and stride length element of the left lower limb except for the right lower limb stride length significantly increased when insoles for postural correction were put one compared to gait with shoes. Speed was highest in the gait element of wearing insoles with postural correction. In addition, according to the result of post hoc test on speed, cadence, and step length, gait with insoles for postural correction obtained more significant result than gait with bare feet and with shoes.

Such increase in gait indexes is because of improvement in balance ability and such change occurs due to high correlation between increase in step length and increase in stride length. Gait evaluation before and after cerebral palsy children's putting on insoles which can support medial longitudinal arch of the foot showed increase in speed and step length like the present study result(21). Decrease in functional gait increases instability during gait resulting from decrease in the support surface or changes in the pressure of the feet. This establishes balance for gait and compensation strategy for stability increase. As a 
result, changes in spatial temporal elements occur and functional elements such as speed and cadence are affected(22). Therefore, insoles for postural correction during gait increase support surface of the feet, increasing stability, and improves balance ability, increasing speed and cadence(23). In addition, putting on increases contact surface of the feet and enhances balance response in a standing posture, thereby decreasing excessive muscular action of the lower limbs and heightening muscle efficiency(24).

Healthy children experience greater change in spatial temporal indexes than cerebral palsy children. According to Holt, the average of cadence is 152 (steps $/ \mathrm{min}$ ) and speed is $99.13(\mathrm{~cm} / \mathrm{s})$ in children before and after school age(25). In the present study as well, when walking in insoles for postural correction, cadence and speed were close to those of healthy children compared to under the other two conditions. In applying gait treatment to cerebral palsy children, gait in shoes was more effective in functional terms than applying barefoot gait training. Research by Holt et al. reached the same conclusion.

Improvement in functional gait is emphasized in order to activities of daily living of spastic cerebral palsy children. Insoles for postural correction will be able to be applied as a useful tool to effectively improve gait speed, cadence, step length, and stride length in cerebral palsy children. This study is a pretest and did not perform kinematic analysis study related to gait cycle. Therefore, future research on barefoot gait, gait in shoes, and gait in insoles for postural correction will be necessary.

\section{CONCLUSION}

This study was conducted in order to present a method to effectively improve gait by analyzing spatial temporal indexes of gait during barefoot gait, gait in shoes, and gait in insoles for postural correction for functional improvement in gait of spastic cerebral palsy children. Spatial temporal indexes of gait were measured using GAITRite system. There was significant difference in speed, cadence, step length, and stride length of the left lower limb among spatial-temporal elements of gait. However, there was no significant difference in stride length of the right lower limb, stance phase time, single support time, double support time, swing phase rate during gait, and stance phase rate during gait among spatial temporal indexes. Gait with insoles for postural correction positively affected functional gait improvement and will be able to be applied to spastic cerebral palsy children.

\section{REFERENCES}

1. Rosenbaum P, Paneth N, Leviton A, et al. A report: the definition and classification of cerebral palsy April 2006. Dev Med Child Neurol Suppl 2007; 109(suppl 109): 8-14.

2. Gage JR, Schwartz MH, Koop SE, et al. The identification and treatment of gait problems in cerebral palsy. John Wiley \& Sons 2009.

3. Wenger DR. The Identification and Treatment of Gait Problems in Cerebral Palsy. Journal of Pediatric Orthopaedics 2010; 30(2): 212.

4. Thompson A, Jarrett L, Lockley L, et al. Clinical management of spasticity. Journal of Neurology, Neurosurgery \& Psychiatry 2005; 76(4): 459-463.

5. Flett PJ. Rehabilitation of spasticity and related problems in childhood cerebral palsy. Journal of Paediatrics and child health 2003; 39(1): 6-14.

6. O'Donnell M. Randomized double-blind placebo controlled trial of the effect of botulinum toxin on walking in cerebral palsy. The Journal of pediatrics 2001; 139(1): 163.

7. Ubhi T, Bhakta B, Ives H, et al. Randomised double blind placebo controlled trial of the effect of botulinum toxin on walking in cerebral palsy. Archives of disease in childhood 2000; 83(6): 481487.

8. Lampe R, Mitternacht J, Schrodl S, et al. Influence of orthopaedic - technical aid on the kinematics and kinetics of the knee joint of patients with neuro-orthopaedic diseases. Brain and Development 2004; 26(4): 219-226.

9. Abel MF, Juhl GA, Vaughan CL, et al. Gait assessment of fixed ankle-foot orthoses in children with spastic diplegia. Archives of physical medicine and rehabilitation 1988; 79(2): 126-133.

10. Neto HP, Grecco LA, Galli M, et al. Comparison of articulated and rigid ankle-foot orthoses in children with cerebral palsy: a systematic review.

11. Pediatric Physical Therapy 2012; 24(4): 308-312. Ferris DP, Sawicki GS, Domingo A. Powered lower limb orthoses for gait rehabilitation. Topics in spinal cord injury rehabilitation 2005; 11(2): 34-49. 
12. Landorf KB, Keenan A-MHerbert RD. Effectiveness of foot orthoses to treat plantar fasciitis: a randomized trial. Archives of internal medicine 2000; 166(12): 1305-1310.

13. Powell M, Seid MSzer IS. Efficacy of custom foot orthotics in improving pain and functional status in children with juvenile idiopathic arthritis: a randomized trial. The Journal of rheumatology 2005; 32(5): 943-950.

14. Kressig RW, Gregor RJ, Oliver A, et al. Temporal and spatial features of gait in older adults transitioning to frailty. Gait Posture 2004; 20(1): 30-35.

15. Scholtes VA, Becher JG, Beelen A, et al. Clinical assessment of spasticity in children with cerebral palsy: a critical review of available instruments. Developmental Medicine \& Child Neurology 2006; 48(1): 64-73.

16. Morris C. A review of the efficacy of lower limb orthoses used for cerebral palsy. Developmental Medicine \& Child Neurology 2002; 44(3): 205-211.

17. Abel MF, Damiano DL. Strategies for increasing walking speed in diplegic cerebral palsy. Journal of Pediatric Orthopaedics 1996; 16(6): 753-758.

18. Grieve D, Gear RJ. The relationships between length of stride, step frequency, time of swing and speed of walking for children and adults. Ergonomics 1966; 9(5): 379-399.

19. Morita S, Yamamoto HK. Gait analysis of hemiplegic patients by measurement of ground reaction force. Scandinavian journal of rehabilitation medicine 1995; 27(1): 37-42.

20. Wang RY, Lin PY, Lee CC, et al. Gait and balance performance improvements attributable to ankle-foot orthosis in subjects with hemiparesis. American journal of physical medicine \& rehabilitation 2007; 86(7): 556-562.

21. Kim SG, Ryu YU, Kim HD. The effect of insole supporting medial longitudinal arch while in spastic cerebral palsy with pes planus. Journal of the Korean Society of Physical Medinice 2012; 7(4): 471-480.

22. Eisenhardt JR, Cook D, Pregler I, et al. Changes in temporal gait characteristics and pressure distribution for bare feet versus various heel heights. Gait Posture 1996; 4(4): 280-286.

23. Crowe A, Samson MM, Hoitsma MJ, et al. The influence of walking speed on parameters of gait symmetry determined from ground reaction forces. Human movement science 1996; 15(3): 347-367.

24. Carison W, Damiano D, Abel M, et al. Biomechanics of orthotic management of gait in spastic diplegia. Gait Posture 1995; 3(2): 102-112.

25. Holt KG, Fonseca ST, LaFiandra ME. The dynamics of gait in children with spastic hemiplegic cerebral palsy: theoretical and clinical implications. Human Movement Science 2000; 19(3): 375-405. 